\title{
CONTROL DIFUSO ADAPTATIVO PARA UN MECANISMO NO LINEAL DE HELICÓPTERO
}

\author{
Juan Paúl Ortiz González ${ }^{1, *}$
}

\section{Resumen}

Este documento presenta el análisis del control por modelo de referencia (FMRLC), que es un método de control adaptivo difuso usado en sistemas no lineales, variantes en el tiempo y difíciles de modelar matemáticamente, que proporciona una metodología para adaptarse al error que se produce con la planta real y el modelo de referencia, el mejor rendimiento se obtiene cuando el error tiende a ser muy pequeño, esta adaptación se consigue moviendo los centros de las funciones de membresía de la base de conocimiento del controlador difuso, de acuerdo con un algoritmo de adaptación. El controlador difuso que se plantea para resolver el problema de control es un controlador difuso proporcional derivativo (PD). La eliminación del error de estado estacionario es gracias a una ganancia integral paralela al controlador FMRLC.

Palabras clave: Control por modelo de referencia (FMRLC), controlador difuso, funciones de pertenencia, proporcional derivativo (PD), error de estado estable.

\begin{abstract}
This paper presents the analyze the adaptive Fuzzy Model Reference Learning Control (FMRLC) is a adaptive fuzzy control method used in nonlinear plants, time-varying and difficult to model them mathematically, which provides a methodology to adapt to the error that occurs with the actual plant and the reference model, the performance is obtained when the error tends to be very small, this adaptation is achieved by moving the centers of the member functions of the knowledge base of fuzzy controller, according to an adaptation algorithm. The fuzzy controller is proposed to solve the control problem is a fuzzy proportional derivative controller (PD). The elimination of steady-state error is thanks to an integral gain FMRLC parallel to the controller.
\end{abstract}

Keywords: Fuzzy model reference learning control (FMRLC), fuzzy controller, member functions, proportional derivative (PD), steady-state error.

\footnotetext{
${ }^{1, *}$ Ingeniero electrónico, Egresado de la Maestría en Control y Automatización Industrial, Universidad Politécnica Salesiana, sede Cuenca. Autor para correspondencia : paul_ortiz02@hotmail.com

Recibido: 04-11-2013, Aprobado tras revisión: 12-11-2013.

Forma sugerida de citación: Ortiz, J. (2013). "Control difuso adaptativo para un mecanismo no lineal de helicóptero". InGENIUS. N. ${ }^{\circ}$ 10, (Julio-Diciembre). pp. 5-11. ISSN: 1390-650X.
} 


\section{Introducción}

Hoy en día, los sistemas difusos son cada vez más robustos por los nuevos mecanismos de adaptación desarrollados con más y más aplicaciones prácticas; este documento toma el algoritmo FMRLC para controlar una planta experimental de helicóptero.

La técnica FMRLC, básicamente, consiste emular el modelo inverso de la planta real para cancelar los cambios generados en el sistema a partir de la obtención de un error y la respuesta deseada de un modelo de referencia planteada por el diseñador del controlador, en donde se establecen tiempos de respuestas y comportamientos. A través de este error el sistema difuso aplica una señal de salida que cambia los centros de las funciones de pertenencia, obteniéndose un mejor control de la planta, debido a que esta técnica busca siempre disminuir el error asegurando que la planta se aproxime cada vez más al modelo de referencia con características tales como: la estabilidad, el máximo sobresalto, el tiempo de establecimiento, etc.

Las funciones de pertenencia utilizadas para el controlador difuso y el modelo inverso son del tipo triangular, las señales de entrada a los bloques difusos son la señal de error y la derivada del error, teniéndose como salidas la señal que actúa sobre la planta y la señal que modifica los centros de las funciones de pertenencia de acuerdo con el algoritmo de adaptación.

La planta usada para la implementación del FMRLC es un modelo de un helicóptero con dos grados de libertad siendo un sistema: múltiple entrada, múltiple salida (MIMO), acoplado y no lineal; debe controlar el ángulo del rotor y el ángulo de guiñada para mantenerlo estable incluso ante perturbaciones externas. Para obtener una respuesta sin error de estado estacionario, se añade una ganancia integral en paralelo al controlador FMRLC garantizando de esta manera, el error cero a lo largo del tiempo.

El presente documento brinda, además, un fundamento referente al diseño de controladores, basados en el método FMRLC utilizando la técnica de aprendizaje basado en reglas bases [1], [2].

\section{FMRLC}

Esta sección es una breve descripción del controlador por modelo de referencia. La estructura fundamental del FMRLC se muestra en la Figura 1. El método FMRLC tiene cuatro partes principales: la planta, el controlador difuso, el modelo de referencia y el mecanismo de aprendizaje. El mecanismo de aprendizaje está conformado por dos partes: el modelo inverso difuso y el modificador de la base de conocimiento. Estas partes se muestran en la Figura 1.

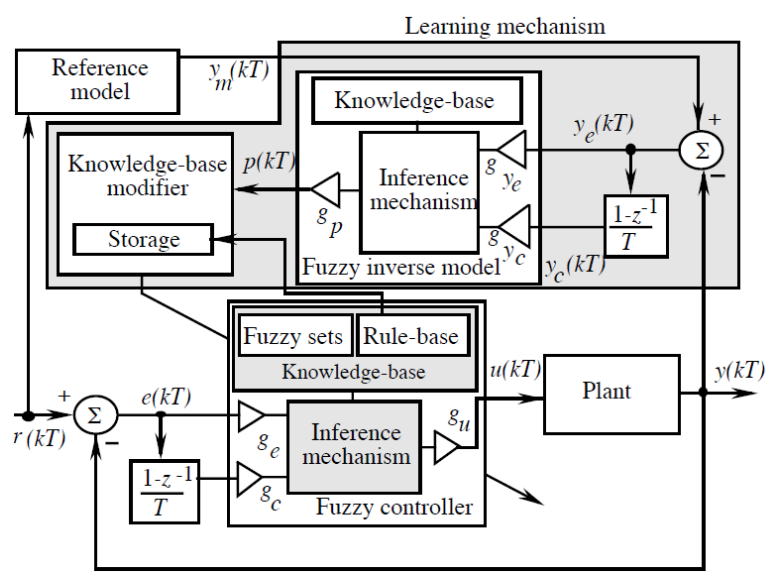

Figura 1. Fuzzy model reference learning controller [3].

Las señales representadas en la Figura 1 son:

- $r(k T)$ referencia del sistema de control difuso.

- $e(k T)$ error entre el valor de la salida del proceso $y(k T)$ y la referencia $r(k T)$.

- $c(k T)$ tasa de cambio de $e(k T)$.

- $u(k T)$ señal de control.

- $y(k T)$ valor de la salida del proceso.

- $y_{m}(k T)$ salida del modelo de referencia cuando la entrada es $r(k T)$.

- $y_{e}(k T)$ error entre $y_{m}(k T)$ y $y(k T)$.

- $y_{c}(k T)$ tasa de cambio de $y_{e}(k T)$.

- $p(k T)$ señal de control para el mecanismo de adaptación.

\subsection{Controlador difuso}

El esquema en la Figura 1, muestra las entradas para el controlador difuso proporcional-derivativo (PD) como son: el error $e(k T)$ y la tasa de cambio del error $c(k T)$.

$$
\begin{gathered}
e(k T)=r(k T)-y(k T) \\
c(k T)=\frac{e(k T)-e(k T-T)}{T}
\end{gathered}
$$

Para este trabajo las señales en la ecuación 1 y ecuación 2 son únicas, por lo que serán tratadas como escalares y no como vectores.

Para un mejor manejo, el universo de discurso para cada entrada, está normalizado al rango de $[-1,+1]$. En esta parte el controlador difuso tiene tres ganancias constantes: $g e, g c$ y $g u$, donde estas constantes son usadas para escalar el universo de discurso de los conjuntos difusos.

La salida $u(k T)$ se obtiene ejecutando los siguientes pasos: 
a) Fusificación de los valores de las entradas $e(k T)$ $\mathrm{y} c(k T)$,

b) Mecanismo de inferencia de acuerdo a la base de reglas $y$,

c) Defusificación para encontrar la salida numérica del controlador difuso. Estas bases de reglas son modificadas por el mecanismo de aprendizaje.

\subsection{Modelo de referencia}

El modelo de referencia puede ser cualquier tipo de sistema dinámico (lineal o no lineal, invariante en el tiempo o variante en el tiempo, tiempo discreto o continuo, etc.) [1]. Esto permite cuantificar el rendimiento deseado del sistema. Las características de diseño del modelo de referencia se eligen según la estabilidad, tiempo de subida, sobreimpulso, etc. [4]. Este modelo toma la entrada de referencia y obtiene la salida deseada.

\subsection{Mecanismo de aprendizaje}

El mecanismo de aprendizaje tiene dos partes: un modelo difuso inverso y un modificador de la bases de conocimiento. El modelo difuso inverso realiza la función de mapeo de los cambios necesarios en la salida del proceso, expresado por $y_{e}(k T)$, a los cambios relativos en las entradas del proceso (denotado por $p(k T))$.

El modificador de la base de conocimiento realiza la función de modificar la base de conocimiento del controlador difuso para producir los cambios necesarios en las entradas del proceso. El modelo inverso y el controlador difuso son similares, con la excepción de la corrección de la base de conocimiento [5] [6] [7].

El objetivo principal del modelo inverso es emular el modelo de la planta para cancelar los efectos dinámicos de la misma [8]. Las entradas del modelo inverso son el error $y_{e}(k T)$ y los cambios del error $y_{c}(k T)$.

$$
\begin{gathered}
y_{e}(k T)=y_{m}(k T)-y(k T) \\
y_{c}(k T)=\frac{y_{e}(k T)-y_{e}(k T-T)}{T}
\end{gathered}
$$

La razón para utilizar el cambio en la salida deseada es proporcionar un poco de "amortiguamiento" en el mecanismo de aprendizaje.

El modificador de la base de conocimiento funciona moviendo los centros de las funciones de pertenencia (MF) del controlador difuso, para llevar a cero el error $y_{e}(k T)$ aproximando sustancialmente la salida real a la salida deseada. El algoritmo para la actualización de los centros es:

$$
\begin{gathered}
c_{n}^{j, \ldots, k, l, \ldots, m}(k T)=c_{n}^{j, \ldots, k, l, \ldots, m}(k T-T)+\ldots \\
\ldots+p_{n}(k T)
\end{gathered}
$$

Más detalles acerca del controlador difuso, el modelo de referencia y el mecanismo de aprendizaje se encuentran en [1], [2], [8].

\subsection{La planta}

La planta Twin Rotor Mimo System (TMRS) mostrada en la Figura 2, es un sistema "múltiples entradas, múltiples salidas". La dinámica acoplada principal característica de la TMRS se muestra en la Figura 3, donde se observa que la acción del ángulo de elevación (pitch angle) influye en cierta medida sobre la salida del ángulo de guiñada (yaw angle) y viceversa.

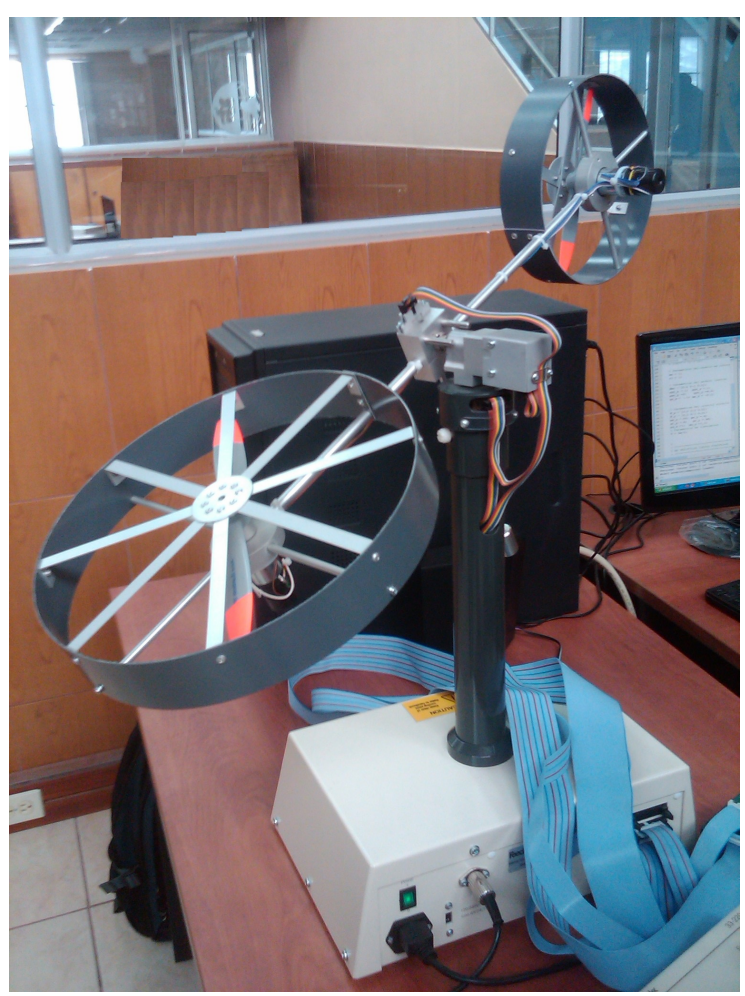

Figura 2. Planta experimental TMRS ${ }^{\odot}$ Feedback Instrument Limited.

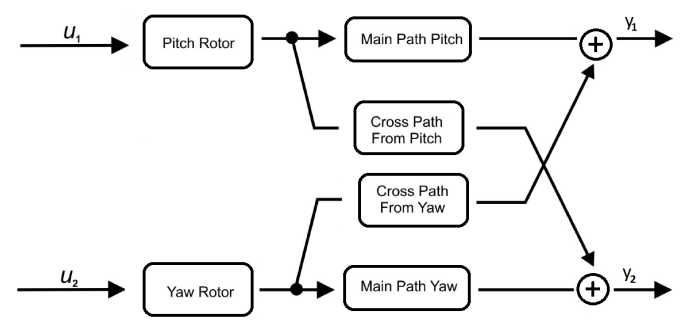

Figura 3. Esquema TMRS $^{\odot}$ Feedback Instrument Limited. 
Los señales de salida de este sistema son el ángulo de elevación $y_{1}$ y el ángulo de guiñada $y_{2}$ medidos en radianes. Las señales de control son $u_{1}$ y $u_{2}$ donde la cota de la señal se establece en $[-2.5 \mathrm{~V} \ldots+2.5 \mathrm{~V}]$.

El control se lleva acabo de forma independiente para cada motor, esto para reducir la complejidad de la base de conocimiento del controlador difuso y de todo el sistema.

\section{Diseño del FMRLC para un mecanismo TMRS}

\subsection{Modelo de referencia}

El modelo de referencia para este caso es:

$$
y_{m}(s)=\frac{\omega_{n}^{2}}{s^{2}+2 \zeta \omega_{n} s+\omega_{n}^{2}}
$$

Donde $\zeta$ y $\omega_{n}$ son el coeficiente de amortiguamiento y la frecuencia natural del sistema deseado. La transformada inversa de Laplace de la ecuación 6 es $\ddot{y}_{m}(t)=$ $\omega_{n}^{2} r(t)-2 \zeta \omega_{n} \dot{y}_{m}(t)-\omega_{n}^{2} y_{m}(t)$ y tomando las variables de estado $x_{1}(t)=y(t)$ y $x_{2}(t)=\dot{y}(t)$ se obtiene las siguientes ecuaciones de espacio de estados:

$$
\begin{gathered}
\dot{x}_{1}(t)=x_{1}(t) \\
\dot{x}_{2}(t)=\omega_{n}^{2} r(t)-2 \zeta \omega_{n} x_{2}(t)-\omega_{n}^{2} x_{1}(t)
\end{gathered}
$$

La ecuaciones 7 y 8 serán usadas para programar el modelo de referencia en la simulación y en la planta experimental mostrada en la Figura 2, con los parámetros del sistema $\omega_{n}=1 \mathrm{rad} / \mathrm{seg}$ y $\zeta=1$ [9], [4], [10].

A continuación se presenta el código en MATLAB ${ }^{\circledR}$ para verificar el modelo de referencia planteado, la Figura 4, muestra su comportamiento.

Código 1. Modelo de referencia.

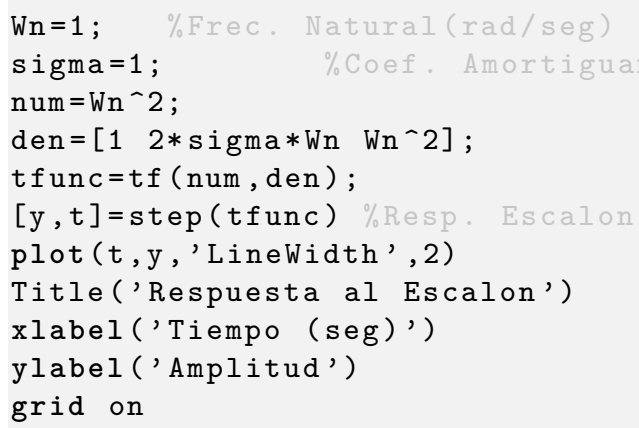

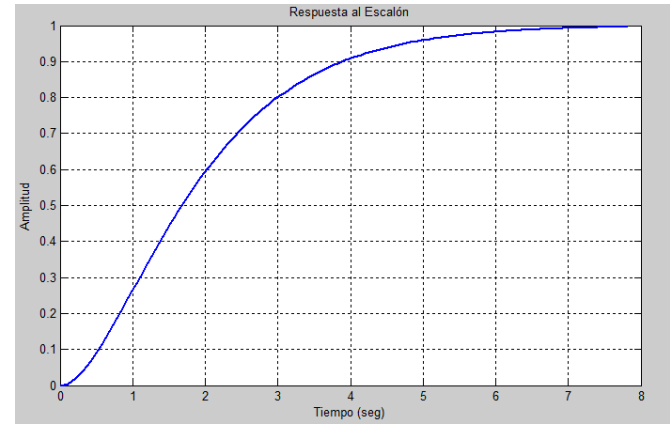

Figura 4. Respuesta al escalón del modelo de referencia.

\subsection{Controlador difuso}

El controlador difuso es implementado con funciones de pertenencia triangulares, el universo de discurso de las entradas y las salidas están normalizadas entre $[-1,+1]$ como se muestra en las Figuras 5 y 6 respectivamente.

Las variables lingüísticas asociadas con cada función de pertenencia son un conjunto de distribución uniforme donde -5 es "negativo muy grande", 0 es "cero" y 5 es "positivo muy grande", en [3] [11] y [12] se amplía la temática sobre la teoría de conjuntos difusos.

La base de reglas del controlador es mostrada en la Tabla 1. Las ganancias de escalado para las entradas del controlador difuso se seleccionaron de la siguiente manera: para el error $\left(g_{e}=2.5\right)$, para la tasa de cambio del error $\left(g_{c}=1\right)$ y para la salida del controlador $\left(g_{u}=0.1\right)$, la ubicación de dichas ganancias se muestran en la Figura 1.

\subsection{Mecanismo de aprendizaje}

Para el mecanismo de aprendizaje la base de conocimiento del modelo inverso será la misma que la del controlador difuso, ya que se tienen entradas y salidas normalizadas, para este caso las constantes son ajustadas $\left(g_{y e}=1\right),\left(g_{y c}=1\right)$ y $\left(g_{p}=0.11\right)$.

El proceso de adaptación de los centros de las funciones de pertenencia está dado por la ecuación 5 .

\section{Simulaciones y resultados}

Las simulaciones del sistema no lineal de la planta TMRS se desarrolla mediante MATLAB ${ }^{\circledR}$ y SIMULINK $^{\circledR}[13]$.

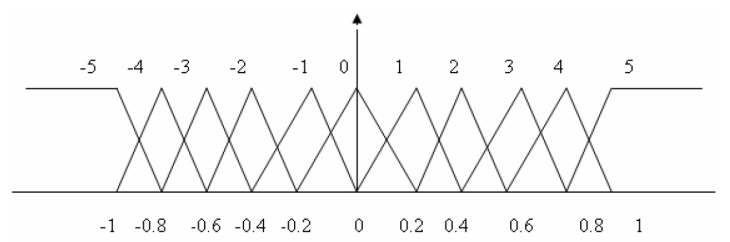

Figura 5. MF para cada entrada, $e(k T)$ y $u(k T)$ [14]. 


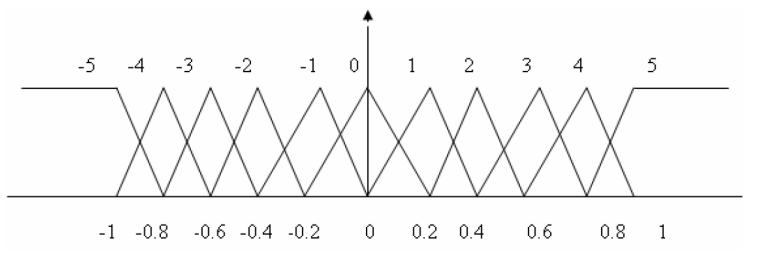

Figura 6. MF para la salida del controlador difuso, $u(k T)[14]$.

\subsection{Simulaciones}

En la Figura 7, se muestra el diagrama de bloques del controlador FMRLC implementado en SimulinK ${ }^{\circledR}$, se puede observar que se añade un factor de acción integral en paralelo al controlador difuso para mejorar el error de estado estable. Para las simulaciones se hace uso de la función de transferencia del mecanismo
TMRS dado por Feedback Instrument Limited ${ }^{\odot}$. El tiempo de muestreo es $1 \mathrm{~ms}$, el diagrama de bloques con los dos controladores FMRLC tanto para el ángulo de elevación como para el ángulo de guiñada se muestra en la Figura 8, adicionalmente se añade al sistemas dos señales del tipo escalón a las salidas del sistema que representan los disturbios.

La Figura 9, presenta la respuesta del sistema, éste posee un tiempo de subida de aproximadamente $5 \mathrm{~s}$ debido a que el modelo de referencia planteado en la Figura 4, forza a que se dé dicha respuesta.

Para poder analizar la estabilidad del sistema simulado se introduce disturbios en el tiempo igual a $40 \mathrm{~s}$ con magnitud 0.3 para ambas señales: pitch y yaw, el controlador compensa dichos disturbios mantenidos a lo largo del tiempo y lleva al error a cero, este efecto se muestra en la Figura 10.

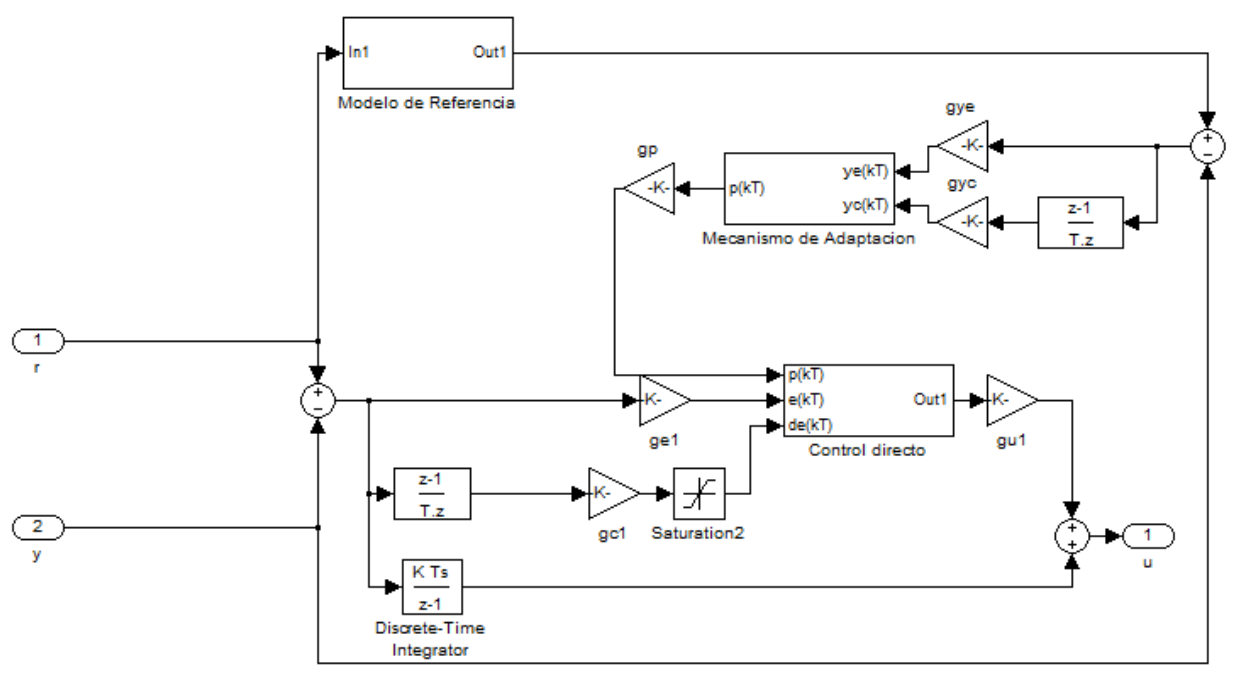

Figura 7. Diagrama de bloques del controlador FMRLC.

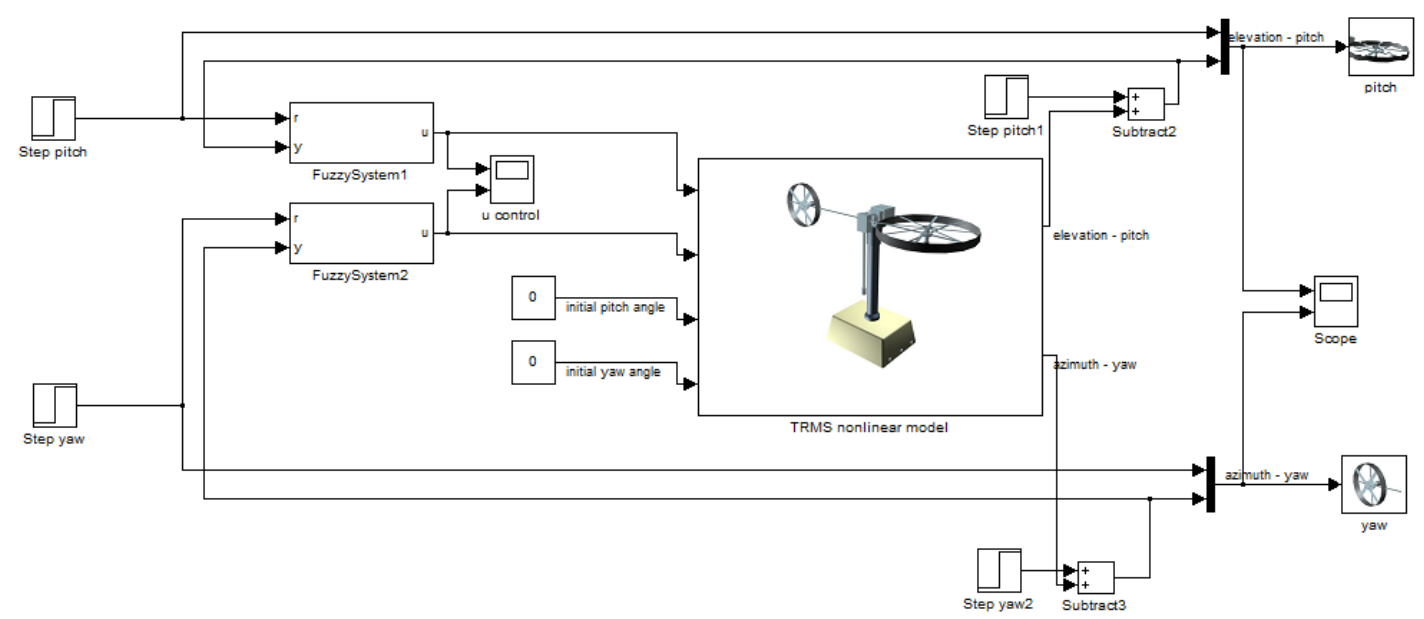

Figura 8. Diagrama de bloques de los controladores FMRLC pitch y yaw, con disturbios en las señales de salida. 
Tabla 1. Reglas de fuzificación.

\begin{tabular}{|c|c|c|c|c|c|c|c|c|c|c|c|c|}
\hline & & \multicolumn{11}{|c|}{$e(k T)$} \\
\hline & & -5 & -4 & -3 & -2 & -1 & 0 & +1 & +2 & +3 & +4 & +5 \\
\hline & -5 & +1.0 & +1.0 & +1.0 & +1.0 & +1.0 & +1.0 & +0.8 & +0.6 & +0.4 & +0.2 & 0.0 \\
\hline & -4 & +1.0 & +1.0 & +1.0 & +1.0 & +1.0 & +0.8 & +0.6 & +0.4 & +0.2 & 0.0 & -0.2 \\
\hline & -3 & +1.0 & +1.0 & +1.0 & +1.0 & +0.8 & +0.6 & +0.4 & +0.2 & 0.0 & -0.2 & -0.4 \\
\hline & -2 & +1.0 & +1.0 & +1.0 & +0.8 & +0.6 & +0.4 & +0.2 & 0.0 & -0.2 & -0.4 & -0.6 \\
\hline & -1 & +1.0 & +1.0 & +0.8 & +0.6 & +0.4 & +0.2 & 0.0 & -0.2 & -0.4 & -0.6 & -0.8 \\
\hline \multirow[t]{6}{*}{$c(k T)$} & 0 & +1.0 & +0.8 & +0.6 & +0.4 & +0.2 & 0.0 & -0.2 & -0.4 & -0.6 & -0.8 & -1.0 \\
\hline & +1 & +0.8 & +0.6 & +0.4 & +0.2 & 0.0 & -0.2 & -0.4 & -0.6 & -0.8 & -1.0 & -1.0 \\
\hline & +2 & +0.6 & +0.4 & +0.2 & 0.0 & -0.2 & -0.4 & -0.6 & -0.8 & -1.0 & -1.0 & -1.0 \\
\hline & +3 & +0.4 & +0.2 & 0.0 & -0.2 & -0.4 & -0.6 & -0.8 & -1.0 & -1.0 & -1.0 & -1.0 \\
\hline & +4 & +0.2 & 0.0 & -0.2 & -0.4 & -0.6 & -0.8 & -1.0 & -1.0 & -1.0 & -1.0 & -1.0 \\
\hline & +5 & 0.0 & -0.2 & -0.4 & -0.6 & -0.8 & -1.0 & -1.0 & -1.0 & -1.0 & -1.0 & -1.0 \\
\hline
\end{tabular}

\subsection{Resultados}

Para obtener los resultados experimentales el controlador diseñado fue implementado en SiMULINK ${ }^{\circledR}$, usando tarjetas de adquisición de datos de Feedback Instrument Limited ${ }^{\odot}$.

Los resultados obtenidos se muestran en la Figura 11, con puntos de referencia del ángulo pitch $0.3 \mathrm{rad}$ y el ángulo yaw de $0.3 \mathrm{rad}$, no se consideraron disturbios y el tiempo de muestreo fue de $1 \mathrm{~ms}$.

En este caso, la planta llega en el tiempo de $5 \mathrm{~s}$ a los valores de referencia y se mantiene la estabilidad del sistema a lo largo del tiempo.

En la Figura 12, se aplican al sistema, disturbios sobre el ángulos pitch en los tiempos $t=28$ y $40 \mathrm{~s}$; y en el ángulo yaw en los tiempos $t=78 \mathrm{~s}$ con amplitud 0.5 , obteniendo como resultado la estabilización del sistema y eliminando el error de estado estable.

Las simulaciones y los resultados obtenidos del controlador permite un seguimiento del modelo de referencia teniendo un tiempo de establecimiento de $5 \mathrm{~s}$ en ambos casos. La estabilidad del mecanismo es fiable y las señales de control acotadas, permitiendo que el funcionamiento sea óptimo.

A pesar de la dinámica altamente acoplada de la planta, el sistema responde correctamente con los dos controladores FMRLC implementados en el mecanismo, la Figura 12, muestra la acción de dichos controladores en sus respectivas variables de control y en los ángulos de salida controlados pitch y yaw.

\section{Conclusiones}

El controlador FMRLC funciona correctamente incluso cuando se aplica a plantas no lineales y variantes en el tiempo, ya que funciona emulando el modelo difuso inverso de la planta para cancelar los efectos dinámicos producido por las no linealidades del mecanismo TMRS.
Las constantes del controlador deben ser calibradas y ajustadas para su correcto funcionamiento, siendo necesario un conocimiento previo de la operación del sistema, otro aspecto importante para el diseño de estos controladores es la normalización del universo de discurso del sistema difuso, ya que el mismo controlador puede ser utilizado para controlar distintas variables como en este caso los ángulos pitch y yaw, simplemente ajustando las constantes para adaptarse al nuevo universo de discurso de la planta que se desee controlar.

El TMRS es un sistema MIMO no variante en el tiempo, los puntos de referencia del rotor y la cola presentan errores de estado estable, los mismos que pueden ser eliminados añadiendo una constante integral.

Se pudo comprobar que el sistema de control trabaja de acuerdo a los parámetros de diseño, incluyendo los disturbios externos, que en ninguno de los casos probados, causó que el sistema perdiera el seguimiento del modelo de referencia o su estabilidad.

Sin embargo, cabe mencionar que en estos sistemas obtener respuestas rápidas del controlador da el éxito de la misión, por lo que se debe tener especial cuidado al ajustar las constantes del sistema para no provocar inestabilidad del mecanismo u obtener sobreimpulsos excesivos en su funcionamiento.

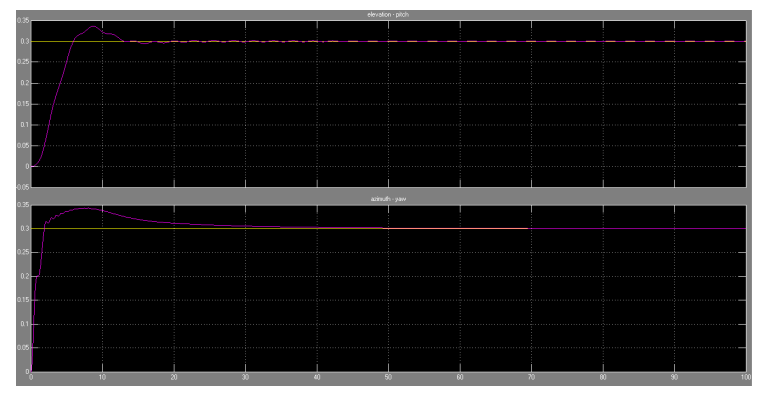

Figura 9. Simulación para el mecanismo TMRS, sin disturbios. 


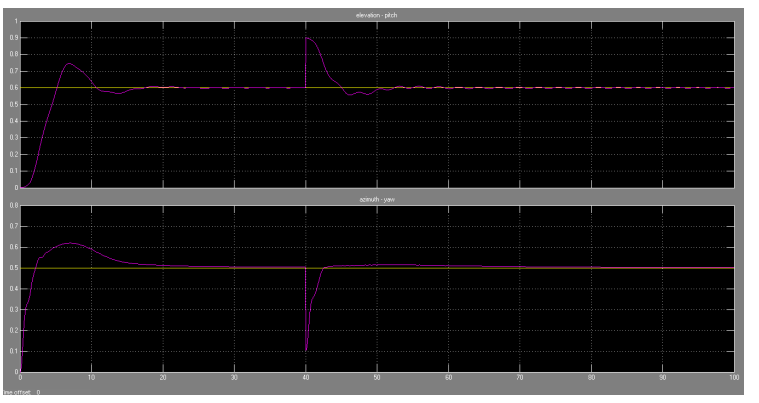

Figura 10. Simulación para el mecanismo TMRS, con disturbios aplicados a las señales pitch y yaw en $\mathrm{t}=40 \mathrm{~s} \mathrm{y}$ amplitud $0.3 \mathrm{rad}$.

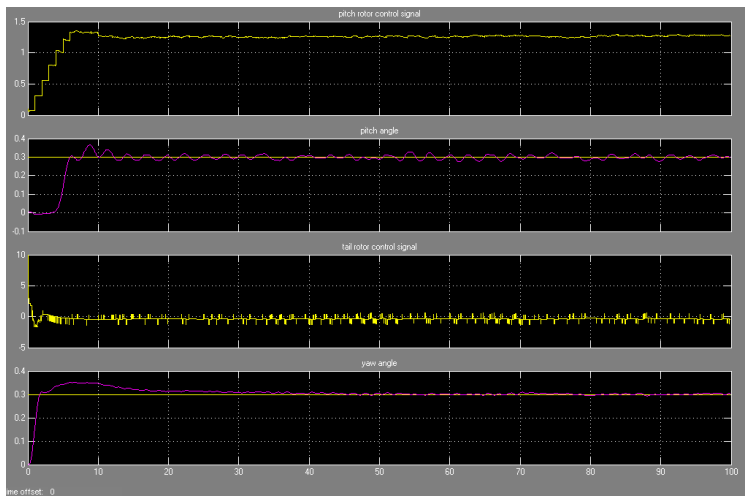

Figura 11. Resultados del mecanismo TMRS sin disturbios.

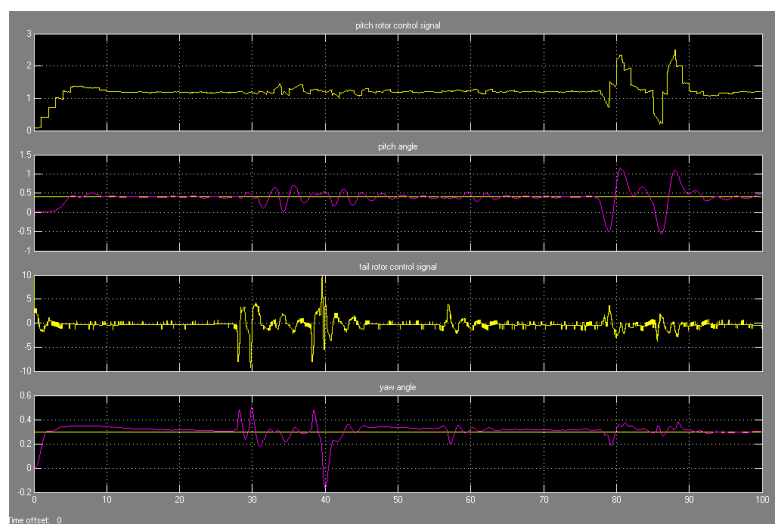

Figura 12. Resultados del mecanismo TMRS con disturbios.

\section{Agradecimientos}

El autor agradece a la Universidad Politécnica Salesiana por haber permitido trabajar en la planta experimental TMRS del Departamento de Control y Automatización, para el desarrollo del presente trabajo.

\section{Referencias}

[1] J. Layne and K. Passino, "Fuzzy model reference learning control for cargo ship steering," Control Systems, IEEE, vol. 13, no. 6, pp. 23-34, 1993.

[2] J. Layne, "Fuzzy model learning control," Master's thesis, Department of Electrical Engineering, The Ohio State University, March 1992.

[3] K. Passino and S. Yurkovich, Fuzzy Control, 1st ed. United States: Addison-Wesley, 1997.

[4] R. C. Dorf, Modern control systems. AddisonWesley Longman Publishing Co., Inc., 1991.

[5] P. Antsaklis and K. Passino, An Introduction to Intelligent and Autonomous Control. Kluwer Academic Publishers, 1993.

[6] J. R. Layne, K. M. Passino, and S. Yurkovich, "Fuzzy learning control for antiskid braking systems," Control Systems Technology, IEEE Transactions on, vol. 1, no. 2, pp. 122-129, 1993.

[7] K. Tanaka and H. O. Wang, Fuzzy control systems design and analysis: a linear matrix inequality approach. Wiley. com, 2004.

[8] N. Abdellatif, Y. Ramdani, and H. Hamdaoui, "A new adaptive controller of facts-based fmrlc aimed at improving power system stability," Serbian Journal of Electrical Engineering, vol. 5, no. 2, pp. 229-245, 2008.

[9] B. Kuo and F. Golnaraghi, Automatic control systems. John Wiley \& Sons New York, 2003, vol. 4 .

[10] K. Ogata, Ingeniería de control moderna 4 ED. Pearson Educación, 2003.

[11] G. J. Klir and B. Yuan, Fuzzy sets and fuzzy logic. Englewood Cliffs, New Jersey: Prentice Hall, 1995.

[12] H. J. Zimmermann, Fuzzy set theory-and its applications, 2nd ed. Springer, 2001.

[13] T. Mathworks, "Fuzzy logic toolbox for use with Matlab," 2006.

[14] P. Khundia and D. Mitra, "Fuzzy model reference learning controller for pitch control system of an aircraft," Georgia Electronic Scientific Journal, no. 3, 2009. 